\title{
A Unified Model of Interstellar Extinction and Polarization ${ }^{1}$
}

\author{
J. Mayo Greenberg and G. Shah \\ Rensselaer Polytechnic Institute \\ Troy, New York
}

$T^{\prime}$

IHE MOST COMPLETELY OBSERVED CHARACTERISTICS OF INTF.RSTELLAR GRAINS are their ability to dim and polarize the light of distant stars in our own galaxy. The principal limitation in previous theoretical models of interstellar dust clouds has been the fact that the extinction and polarization have been calculated for different particles, the connection between these types of particles being semiquantitative at best.

A knowledge of the electromagnetic scattering properties of the various proposed grains is a fundamental ingredient of any theory of extinction and polarization. All the types of grains which have been suggested, except those of reference 1, scatter electromagnetic radiation by a classical process, i.e., a process which is characterized by a classical application of Maxwell's equations and for which the particles are defined by a size, shape, and index of refraction. In spite of the fact that the methods of application of the classical theory are completely understood, numerical results for the particular range of application which appears to be needed in the interstellar dust problem have been somewhat limited.

Most of the previous calculations of extinction have been performed on spherical particles of various types: dielectric (dirty ice), metallic, graphite, and core-mantle (graphite plus dirty ice or metal plus dirty ice). Regardless of whether one tries to fit the observed extinction curves with a single size of particle or a distribution of sizes, one arrives at a characteristic size depending on the particular optical properties chosen for the grains. In all cases the particle dimension is such that incomplete numerical (analytical or experimental) results were available for the extinction cross sections of arbitrarily oriented nonspherical particles of the appropriate dimension. For example, the only really quantitative

\footnotetext{
1 The contents of this paper were published previously in the Astrophys. J. (pub. by the Univ. of Chicago Press), vol. 145, 1966, p. 63.
} 


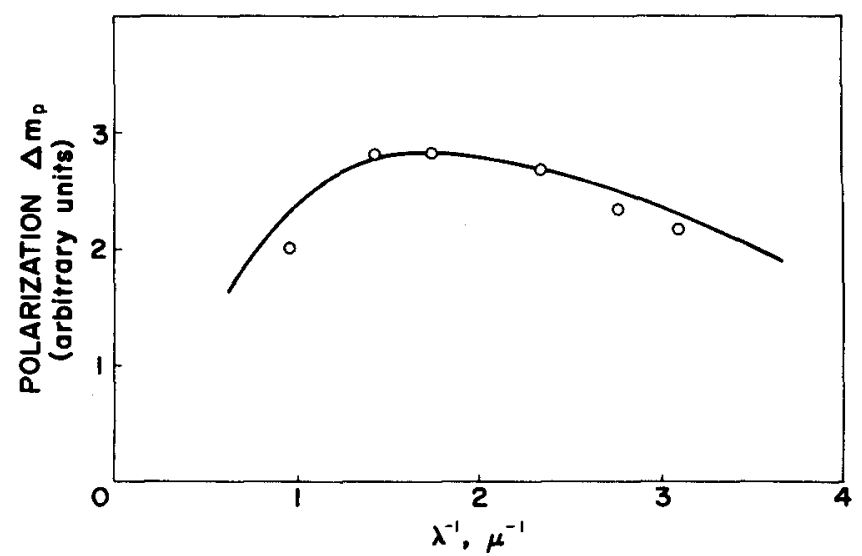

FIGURE 1.-Observed percentage polarization (each point is average for eight stars) as a function of inverse wavelength. (See ref. 3.) Solid curve is calculated for perfectly oriented dielectric (index of refraction $=1.70$ ) cylinders with a distribution of radii according to an Oort-van de Hulst size distribution which, for spheres, reproduces a standard extinction curve.

calculations of the wavelength dependence of polarization could be made for perfectly alined circular cylinders. By choosing a distribution of radii for dielectric cylinders which is the same as that for the spheres which give a good representation of the extinction, it was indeed possible to achieve a good fit to the observed wavelength dependence of polarization. (See ref. 2 and figs. 1 and 2.) Semiquantitative calculations have also been made on the polarization -its amount and wavelength dependence-produced by dielectric spheroidal particles. The results are given in reference 4 . However, in no case has it been possible to

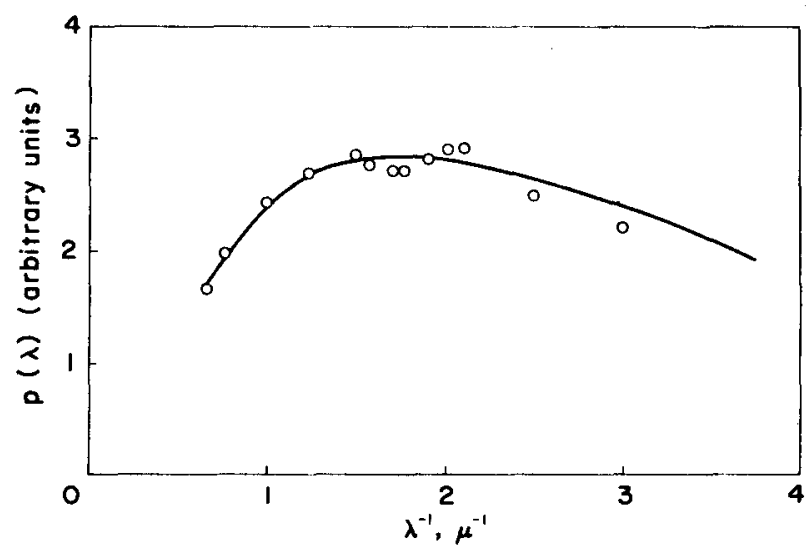

Figure 2. - Comparison between theoretical wavelength dependence of polarization produced by a distribution of sizes of cylinders (solid curve) with that produced by a single size cylinder of radius $a=0.20 \mu$ (open circles). 
perform a simultaneous and equally quantitative calculation of extinction and polarization.

A complete computing scheme, equivalent to the Mie theory for spheres, for the scattering by arbitrarily oriented infinite cylinders is given in references 5 and 6 . These new schemes have made it possible to saiculate simultaneously the extinction and polarization by realistically oriented particles. The justification for the infinite cylinder model lies in the fact that the extinction and polarization by infinite cylinders differ quantitatively but not qualitatively from the extinction and polarization produced by finite elongated particles. (See ref. 4.) Furthermore, if the grains are indeed needlelike in structure or perhaps consist of loose agglomerations of needles, this representation would be fully realistic.

In this paper we consider particles whose index of refraction $m$ is 1.33 , and the application, therefore, is most reliably made to the wavelength region where icelike materials are nonabsorbing; namely, $1 \leqq \lambda^{-1} \leqslant 3 \mu^{-1}$. The orientation is treated from the point of view of the Davis-Greenstein mechanism (ref. 7).

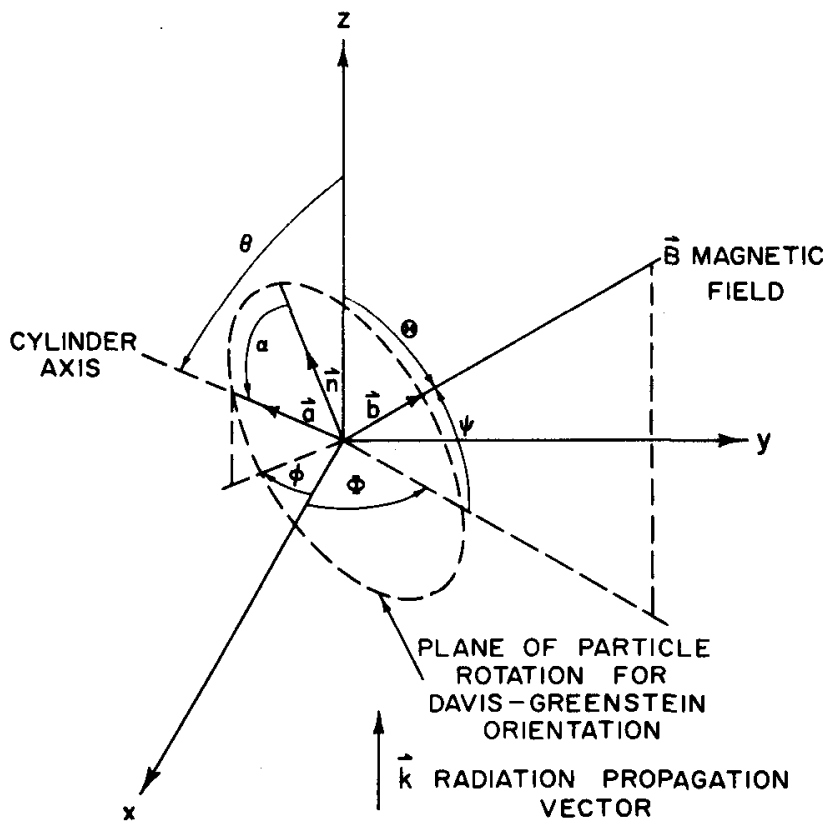

FIGURE 3.-Geometrical configuration for axially symmetric elongated particle spinning about its short axis which is alined with the magnetic field direction. Radiation is propagated along $z$-direction. 


\section{GENERAL FORMULATION FOR EXTINCTION AND POLARIZATION BY AXIALLY SYMMETRIC PARTICLES}

The extinction and polarization are determined by the optical depths $\tau_{x}$ and $\tau_{y}$ corresponding to radiation polarized along appropriate rotated $x$ - and $y$-directions and propagated along the $z$-direction. (See fig. 3.) For axially symmetric particles the most general form for these optical depths is given by (see ref. 2)

$$
\begin{aligned}
\tau_{x, y}=\int_{0}^{L} \mathrm{~d} z \int_{0}^{\infty} \mathrm{d} a \int_{0}^{\infty} \mathrm{d} e \int_{0}^{\pi} \sin \theta & \mathrm{d} \theta \int_{0}^{2 \pi} \mathrm{l} \phi n(z, a, e) \\
\times & \times f(z, \theta, \phi, a, e) C_{x, y}(\theta, \phi, a, e, \lambda)
\end{aligned}
$$

where

$$
\begin{aligned}
& L \quad \text { total path length } \\
& \text { a a characteristic linear dimension } \\
& e \quad \text { ratio of length to width } \\
& \lambda \text { wavelength of radiation } \\
& C_{x}(\theta, \phi, a, e, \lambda) \quad \text { extinction cross section } \\
& C_{y}(\theta, \phi, a, e, \lambda) \quad \text { extinction cross section } \\
& n(z, a, e) \quad \text { size distribution function } \\
& f(z, \theta, \phi, a, e) \quad \text { angular distribution function }
\end{aligned}
$$

The extinction is related approximately to the optical depths by

$$
\Delta m=1.086 \frac{\tau_{x}+\tau_{y}}{2}
$$

where the approximation is based on the fact that, in practice, the ratio of polarization to extinction is considerably less than 1 .

The polarization is given exactly by

$$
\Delta m_{p}=1.086\left(\tau_{x}-\tau_{y}\right)_{\max }
$$

where the subscript "max" denotes that $\tau_{x}$ represents the maximum optical depth and $\tau_{y}$, the minimum optical depth as the plane of the analyzer is rotated.

Although it is now within the realm of possibility to consider the fully general form of equation (1), it is probably more instructive, and certainly simpler to visualize, if we consider its implications in gradually increasing complexity. 
Throughout this paper, unless otherwise stated, the integrations over $z, a$, and $e$ are ignored. This reduces equation (l) to the form

$$
\tau_{x, y}=\int_{0}^{\pi} \int_{0}^{2 \pi} \mathrm{d} \phi \sin \theta \mathrm{d} \theta f(\theta, \phi) C_{x, y}(\theta, \phi)
$$

Insofar as infinite cylinders are concerned, it is only the elongation factor which needs to be suppressed. The simplest orientation is that in which all particles are lined up in the same direction. To denote such perfect alinement the letters P.F. (picket fence) are used and all the particles are assumed to point along the $x$-direction:

$$
f_{\mathrm{P} . \mathrm{F} .}(z, \theta, \phi, a, e)=\delta(\cos \theta) \delta(\phi)
$$

where $\delta$ is the Dirac delta function.

For perfect Davis-Greenstein orientation the particles all spin with their short axes along the direction of the magnetic field. In figure 3 , $B$ is defined by the angles $\Theta$ and $\Phi$ with respect to the $z$ - and $x$-axes. If $b$ is a unit vector along $B$ and $a$ is a unit vector along the particle symmetry axis, then the plane of rotation of the axis is defined by $\mathbf{a} \cdot \mathbf{b}=\mathbf{0}$. If the symmetry axis makes the angles $\Theta$ and $\phi$ with respect to the $x y z$ frame and the angle $a$ with respect to some fixed direction $n$ in its plane of rotation, we may write

$$
\mathbf{b} \cdot \mathbf{n}=0, \quad \mathbf{a} \cdot \mathbf{n}=\cos a, \quad \mathbf{n} \cdot \hat{\mathbf{z}}=\sin \Theta,
$$

where $\hat{\mathbf{z}}$ is a unit vector along the $z$-axis.

The appropriate vector relations yield

$$
\begin{aligned}
& \cos \theta=\sin \Theta \cos a \\
& \cos \phi=\frac{\cos \Theta \cos \Phi \cos a-\sin \Phi \sin a}{\cos \Theta \sin \Phi \cos a+\cos \Phi \sin a} .
\end{aligned}
$$

For any axially symmetric particle, the cross sections for arbitrary orientations with respect to the direction and state of polarization of the incident radiation can be derived from two basic cross sections, $C_{E}(\Theta)$ and $C_{H}(\Theta)$, where $\Theta$ is the angle between the incident radiation and the symmetry axis. The cross section $C_{E}$ is that for which the electric vector of the radiation is in the plane containing the direction of propagation and the symmetry axis. The cross section $C_{H}$ is similarly defined for the magnetic vector of the radiation. For the configuration in figure 3 , with incident unpolarized radiations, it can be shown that

$$
\begin{aligned}
& C_{x}=C_{E}(\theta) \cos ^{2} \phi+C_{H}(\theta) \sin ^{2} \phi \\
& C_{y}=C_{E}(\theta) \sin ^{2} \phi+C_{H}(\theta) \cos ^{2} \phi
\end{aligned}
$$


The extinction and polarization are derived from the sum and difference of these two quantities given by

$$
\begin{aligned}
C_{x}+C_{y} & =C_{E}(\theta)+C_{H}(\theta) \\
C_{x}-C_{y} & =\left[C_{E}(\theta)-C_{H}(\theta)\right] \cos 2 \phi
\end{aligned}
$$

Substitution of equations (9) and (10) into equation (4) and use of the appropriate forms for the angular distribution function $f$ yields

Perfect
alinement $\left\{\begin{array}{l}\frac{2}{1.086}(\Delta m)_{\text {P.F. }}=C_{E}\left(\frac{\pi}{2}\right)+C_{H}\left(\frac{\pi}{2}\right) \\ \frac{1}{1.086}\left(\Delta m_{p}\right)_{\text {P.F. }}=C_{E}\left(\frac{\pi}{2}\right)-C_{H}\left(\frac{\pi}{2}\right)\end{array}\right.$

$\begin{gathered}\text { Perfect Davis- } \\ \text { Greenstein } \\ \text { alinement }\end{gathered}$$\left\{\begin{array}{l}\frac{2}{1.086}(\Delta m)_{\text {D.G. }}=\frac{1}{\pi} \int_{0}^{\pi} \mathrm{d} \alpha\left[C_{E}(\theta)+C_{H}(\theta)\right] \\ \left.=\left\{\frac{1}{\pi} \int_{0}^{\pi} \mathrm{d} \alpha\left[C_{E}\right)_{\text {D.G. }}(\theta)-C_{H}(\theta)\right] \cos 2 \phi\right\}_{\max }\end{array}\right.$

where $\theta$ and $\phi$ are defined by equations (5) and (6).

The maximum value of the integrand in equation (14) is, for symmetry reasons, obviously obtained for $\Phi=\pi / 2$ (B in the $y z$-plane in fig. 3). Under more general conditions, such as, e.g., would hold when the radiation passes through several clouds in each of which the orientation is different, it is convenient to define for each cloud the quantities

$$
\begin{aligned}
& C=\frac{1}{\pi} \int_{0}^{\pi} \mathrm{d} \alpha\left[C_{E}(\theta)-C_{H}(\theta)\right] \cos 2 \phi, \\
& S=\frac{1}{\pi} \int_{0}^{\pi} \mathrm{d} \alpha\left[C_{E}(\theta)-C_{H}(\theta)\right] \sin 2 \phi,
\end{aligned}
$$

in terms of which the polarization due to a single cloud would be given by

$$
(1.086)^{-1}\left(\Delta m_{p}\right)_{\text {D.G. }}=\left(C^{2}+S^{2}\right)^{1 / 2}
$$

and the polarization due to several clouds is determined by summing the quantities over all clouds involved. 


\section{EXTINCTION CROSS SECTIONS FOR SMALL SPHEROIDS AND FOR INFINITE CYLINDERS}

For very small particles satisfying the condition for validity of the Rayleigh approximation $[(2 \pi a) / \lambda \ll 1]$ the direction of the electric field of the incident radiation relative to the particle completely defines the cross section independent of the direction of the radiation. For an axially symmetric particle $C_{||}$and $C_{\perp}$ are defined as the extinction cross sections when the electric vector is parallel to the symmetry axis and perpendicular to the symmetry axis, respectively. For spheroids, the formulas for $C_{\|}$and $C_{\perp}$ may readily be obtained, for example, from reference 8.

For arbitrary orientation,

$$
\begin{aligned}
C_{E}(\theta) & =C_{\|} \sin ^{2} \theta+C_{\perp} \cos ^{2} \theta \\
C_{H}(\theta) & =C_{\perp} \\
C_{E}+C_{H} & =\left(C_{\|}+C_{\perp}\right)+\left(C_{\perp}-C_{\|}\right) \cos ^{2} \theta \\
C_{E}-C_{H} & =\left(C_{\|}-C_{\perp}\right) \sin ^{2} \cdot \theta
\end{aligned}
$$

The basic cross sections for infinite cylinders are obtained from equations analogous to, but somewhat more complicated than, the ones used for computing cross sections of spheres. The pertinent equations used in computing the cylinder cross sections are summarized in the following equations. (Note that these cross sections are normalized to be cross sections per unit length.)

$$
\begin{aligned}
& C_{E}(\theta)=\frac{4}{k} \operatorname{Re}\left[b_{0}^{E}(\theta)+2 \sum_{n=1}^{\infty} b_{n}^{E}(\theta)\right] \\
& C_{H}(\theta)=\frac{4}{k} \operatorname{Re}\left[a_{0}^{H}(\theta)+2 \sum_{n=1}^{\infty} a_{n}^{H}(\theta)\right]
\end{aligned}
$$

where

$$
\begin{gathered}
b_{n}^{E}(\theta)=R_{n} \frac{A_{n}(\mu) B_{n}(\epsilon)-n^{2} S^{2} \cos ^{2} \theta}{A_{n}(\epsilon) A_{n}(\mu)-n^{2} S^{2} \cos ^{2} \theta} \\
a_{n}^{H}(\theta)=R_{n} \frac{A_{n}(\epsilon) B_{n}(\mu)-n^{2} S^{2} \cos ^{2} \theta}{A_{n}(\epsilon) A_{n}(\mu)-n^{2} S^{2} \cos ^{2} \theta} \\
R_{n}=J_{n}(l a) / H_{n}(l a) \\
A_{n}(\xi)=\frac{H_{n}^{\prime}(l a)}{l a H_{n}(l a)}-\xi \frac{J_{n}^{\prime}\left(l_{1} a\right)}{l_{1} a J_{n}\left(l_{1} a\right)} \\
B_{n}(\xi)=\frac{J_{n}^{\prime}(l a)}{l a J_{n}(l a)}-\xi \frac{J_{n}^{\prime}\left(l_{1} a\right)}{l_{1} a J_{n}\left(l_{1} a\right)} \\
271-992 O_{-67-11}
\end{gathered}
$$




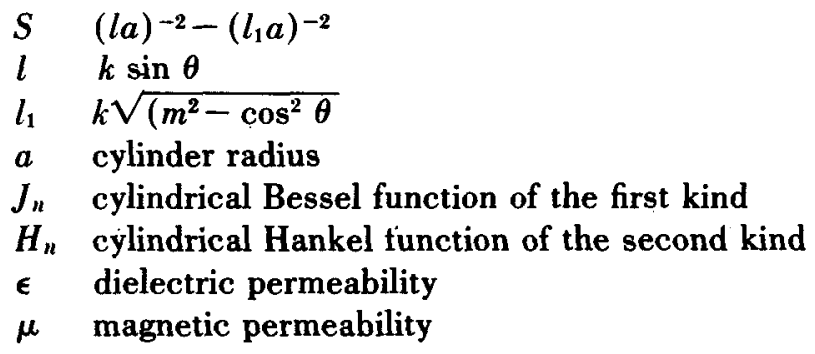

In the present calculations, restricted to nonabsorbing, nonmagnetic particles, $\epsilon=m^{2}$ and $\mu=1$. Some sample results of the computations of equations (22) and (23) are presented in figures 4 to 6 as extinction efficiencies (i.e., cross sections per unit area; $Q=C / 2 a$ ). Figure 4 contains the well-known curves for the variation of extinction efficiency with wavelength for an infinite cylinder whose axis is perpendicular to the direction of the incident radiation. Figures 5 and 6 demonstrate the effect of obliqueness on the extinction efficiencies over a range of selected values of $k a$. With regard to subsequent application to the problem of interstellar extinction and polarization, the most obviously interesting results are: (1) the average polarizing ability of cylinders, as exhibited by the average of $Q_{E}-Q_{H}$ with angle $\theta$, undergoes the greatest reduction relative to its maximum value at $\theta=\pi / 2$ for small values of $k a$ (long wavelength); and (2) the average value of $Q_{E}+Q_{H}$, relative to its value at $\theta=\pi / 2$, tends to decrease more or less uniformly with increasing $k a$.

\section{EXTINCTION AND POLARIZATION RESULTS}

The various polarization and extinction measures for the very small Rayleigh particles can be analytically obtained. Substituting equations (20) and (21) into equations (11) and (14), performing the required integration, and denoting the appropriate quantities by the superscript $R$ (for Rayleigh), yield

Perfect
alinement $\left\{\begin{array}{c}\frac{2}{1.086}(\Delta m)_{\mathrm{P} . \mathrm{F} .}^{R} \\ \frac{1}{1.086}(\Delta m)_{\mathrm{P} . \mathrm{F} .}^{R} \mid=C_{\|}+C_{\perp}, \\ \left(\frac{\Delta m_{p}}{\Delta m}\right)_{\mathrm{P} . \mathrm{F} .}^{R}=2 \frac{C_{\|}-C_{\perp}}{C_{\|}+C_{\perp}} ;\end{array}\right.$ 


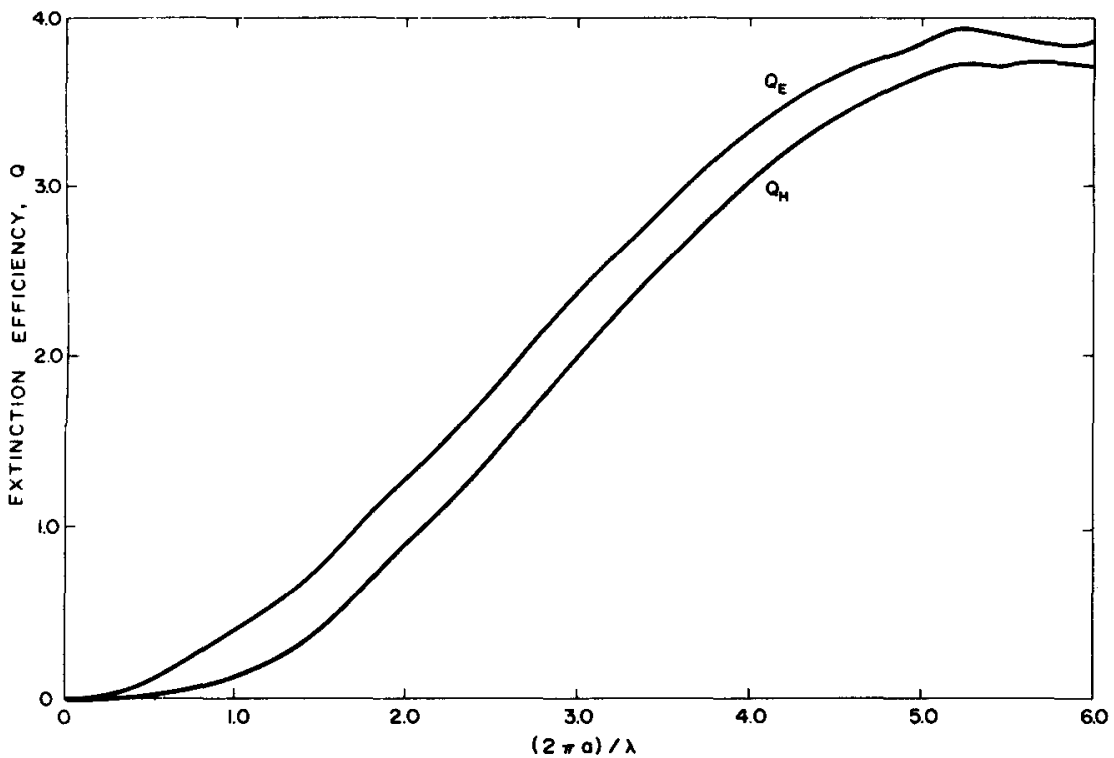

FIGURE 4. - Extinction efficiencies as a function of $k a=2 \pi a / \lambda$ for infinite dielectric $(m$ $=1.33$ ) circular cylinder whose axis is perpendicular to direction of propagation of radiation. Subscripts $E$ and $H$ refer, respectively, to the cases in which $E$ and $H$ are parallel to the cylinder axis.

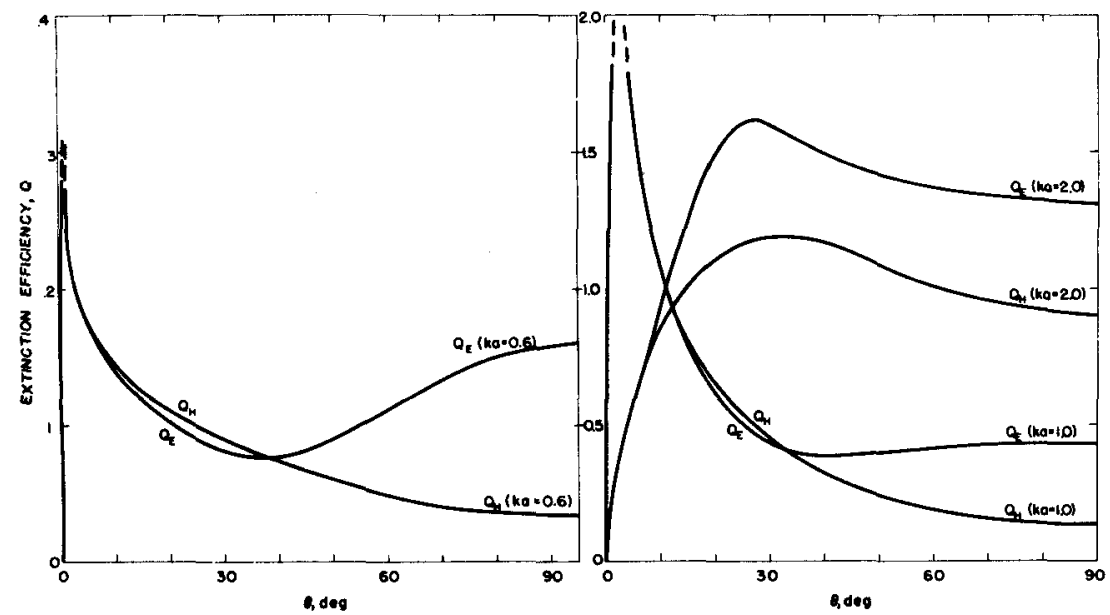

Figure 5. - Extinction efficiencies as a function of orientation angle for infinite dielectric $(m=1.33)$ circular cylinders. Normal incidence is given by $\theta=90^{\circ}$. The peak value of $Q$ for $k a=0.6$ is 3.33 , and for $k a=1.0$, it is 3.1. Note the crossing of the curves for $Q_{E}$ and $Q_{H}$ (and consequent polarization reversal) as the obliqueness increases ( $\theta$ decreasing). 


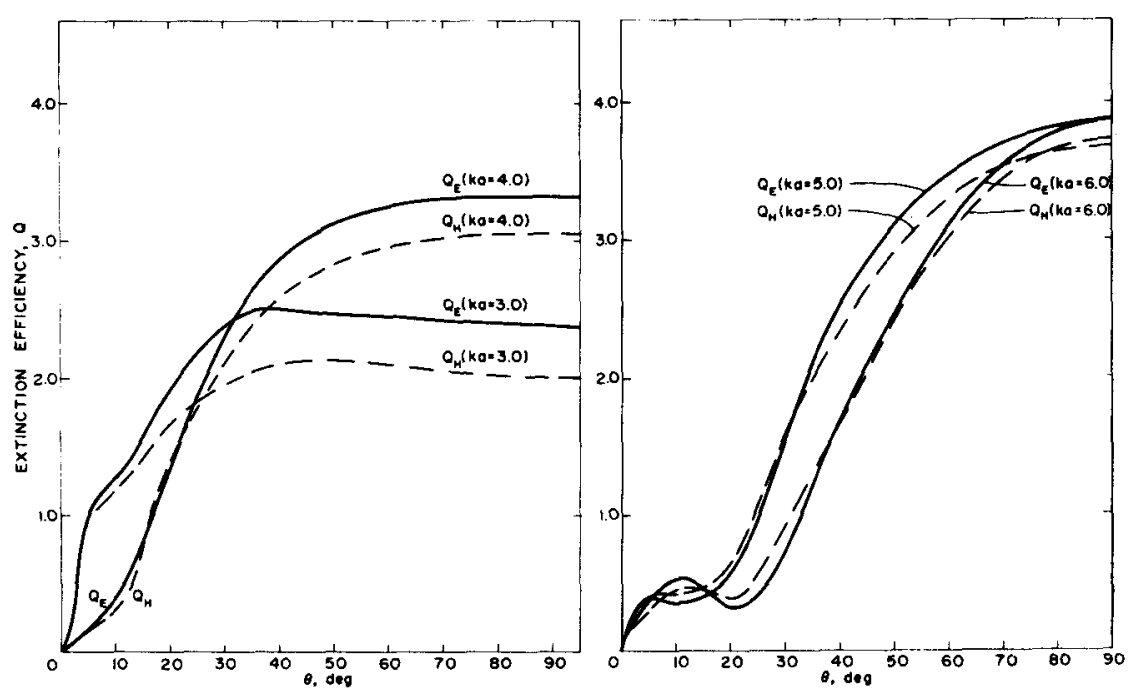

FIGURE 6. - Extinction efficiencies for infinite dielectric $(m=1.33)$ circular cylinders as a function of orientation angle. Normal incidence is given by $\theta=90^{\circ}$. Note the several crossings of the curves for $Q_{E}$ and $Q_{H}$ (representing polarization reversals).

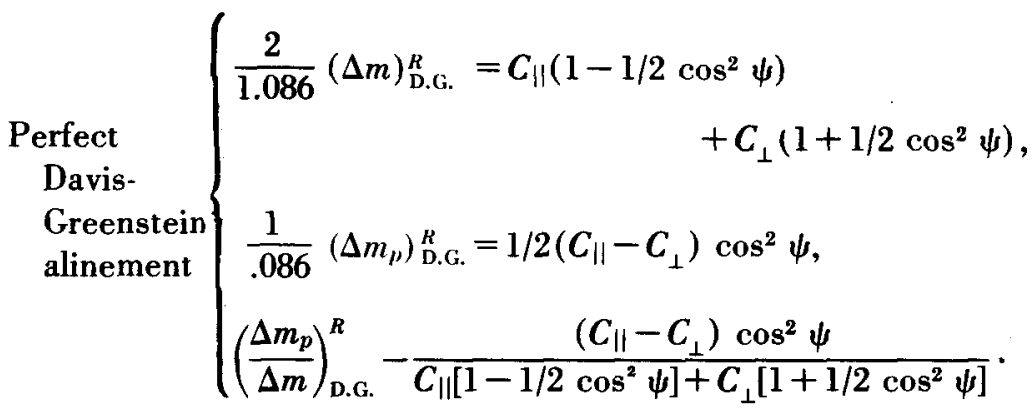

The analogous results for infinite cylinders whose index of refraction is $m=1.33$ are presented in figures 7 and 8 . These curves (except for the uppermost one in fig. 8) are obtained by numerically evaluating the cross sections defined in equations (22) and (23), substituting these values into equations (13) and (14), respectively, and finally evaluating these expressions by numerical integrations. The uppermost curve in figure 8 is the wavelength dependence of polarization if the cylinders are in picket-fence alinement and is obtained by taking the difference between the extinction efficiencies $Q_{E}$ and $Q_{H}$. as given in figure 4.

It is readily seen by comparing the results of figures 7 and 8 with the observed extinction and polarization curves that, even for a single size particle (all cylinders of the same radius), there is a good resemblance between theory and observation. The scale to be chosen corresponds 


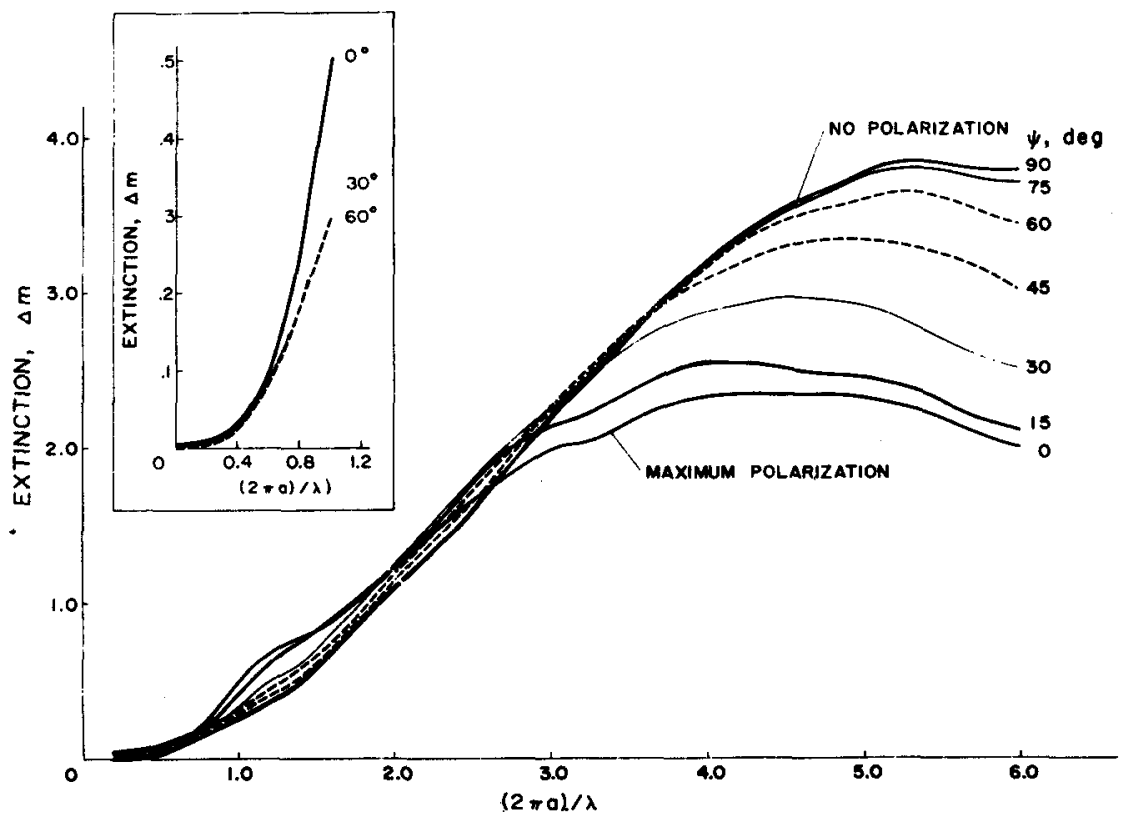

FIGURE 7.-Variation with $k a=(2 \pi a) / \lambda$ of extinction produced by infinite dielectric cylinders spinning in planes which make various angles $\psi$ with respect to direction of incident radiation. The angle $\psi=0^{\circ}$ corresponds to the case in which the spinning plane contains the propagation vector and thus gives rise to the maximum degree of polarization.

roughly to associating an inverse wavelength, $\lambda^{-1}=1$, with a value of $k a$ in the range $1.1 \leqslant k a \leqslant 1.25$. In view of the fact that the calculations are for a pure real index of refraction as well as for a single size of particle, only qualitative inferences can be made particularly in the infrared and ultraviolet where dielectric particles may be expected to be absorbing.

In the range of $k a$ (or $\left.\lambda^{-1}\right)$ corresponding to high polarization $(2 \leqslant k a \leqslant 3$ in fig. 8) it is interesting to note that all the extinction curves in figure 7 are similar in magnitude and that, as a consequence, the ratio of polarization to extinction varies almost entirely according to the amount of polarization. The oscillations in the polarization curves occur because only one size of particle is considered. These oscillations will certainly disappear when a distribution of sizes is considered.

It is instructive at this point to compare the results for small particles with those for cylinders insofar as the amount of polarization depends on the kind of orientation. According to equations (26) and (29), one would predict that the ratio of polarization to extinction would drop by a factor of $1 / 2$ (approximately - assuming that the ratio $C_{\|} / C_{\perp}$ is not much larger than 1) in going from picket-fence alinement to perfect Davis-Greenstein orientation ( $B$ perpendicular to line of sight, $\psi=0^{\circ}$ ); 
however, as one sees in figure 8 , the reduction is only by a factor of about $3 / 4$. A previous estimate of the reduction factor as being about 0.6 was made in reference 4 for spheroids of elongation 2 .

According to figures 7 and 8 , the obvious consequences of decreasing the angle between the magnetic field and the line of sight (increasing $\psi$ ) are: (1) reducing the amount of polarization; (2) broadening of the wavelength dependence of polarization; and (3) increasing extinction in the ultraviolet relative to the visible (assuming a reasonable scale of $\lambda^{-1}$ relative to $k a$ ). Item (3) has already been predicted in references 9 and 10 on the basis of various approximations. The advantage of the present calculation is that one may hope to correlate not only the amount but also the wavelength dependence of polarization with variations of the wavelength dependence of extinction, particularly in the ultraviolet.

The effect of reducing the amount of polarization (item (1)) may be examined and compared with the equivalent effect for small particles by considering equation (28) (assuming as before that the denominator of eq. (29) varies only slightly with $\psi$ ). The Rayleigh approximation predicts that, as the magnetic field makes an angle $\pi / 2-\psi$ with respect to the line of sight, the polarization is reduced by the factor $\cos ^{2} \psi$.

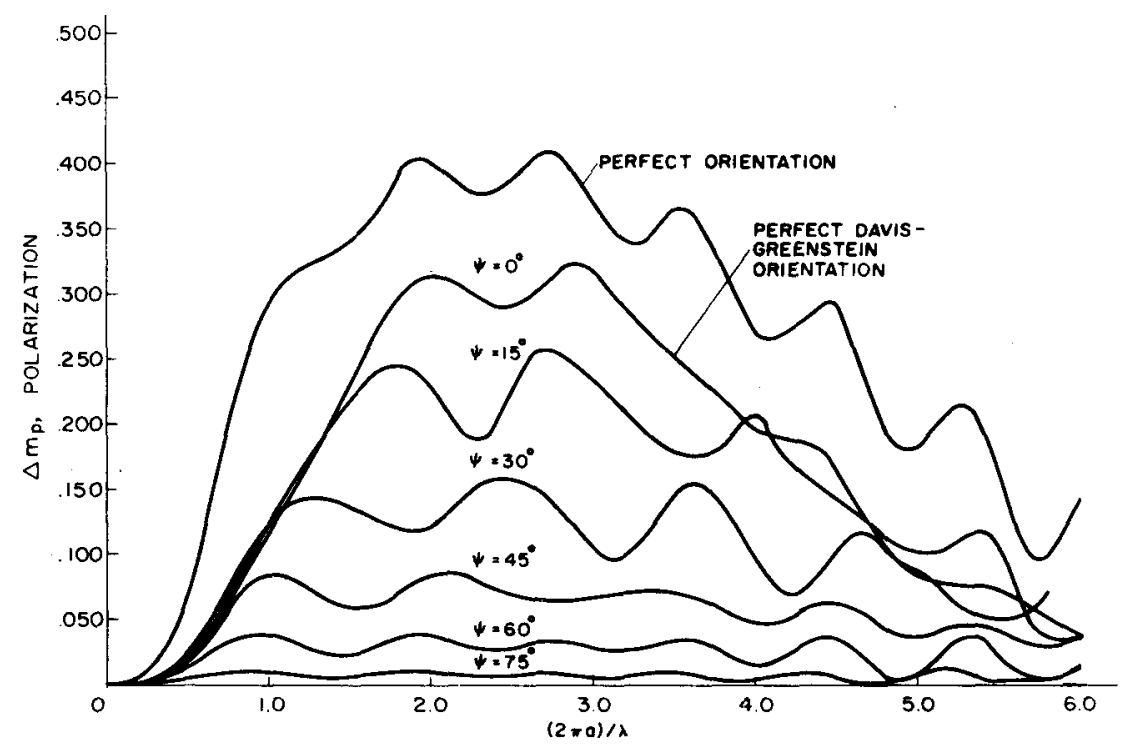

Figure 8. - The uppermost curve is the variation of polarization with $k a=(2 \pi a) / \lambda$ for perfectly alined infinite dielectric cylinders $\left(Q_{E}-Q_{H}\right.$ in figure 4). The other curves give the variation of the wavelength dependence of polarization produced by cylinders spinning in planes which make various angles $\psi$ with respect to the direction of incident radiation. The case $\psi=0^{\circ}$ corresponds to the case in which the spinning plane contains the propagation vector. The angle $90^{\circ}-\psi$ is the angle between the magnetic field and the direction of light propagation. 
TABle I.-Dependence of Amount of Polarization on Magnetic-Field Direction

\begin{tabular}{|c|c|c|c|}
\hline$\psi, \operatorname{deg}$ & $\cos ^{2} \psi$ & $\begin{array}{r}{\left[\left(\Delta m_{p}\right)_{\phi} /\left(\Delta m_{p}\right)_{0}\right] /} \\
\cos ^{2} \psi^{\mathrm{a}}\end{array}$ & ,a, $.\left(\Delta m_{p}\right)_{\psi} /(\Delta m)$ \\
\hline & & & \\
0 & 1 & 1 & 0.16 \\
30 & 0.93 & 0.9 & .13 \\
45 & .75 & .7 & .08 \\
60 & .5 & .5 & .04 \\
\hline
\end{tabular}

${ }^{a}$ Values of $\left(\Delta m_{p}\right)_{\psi}$ and $\left(\Delta m_{p}\right)_{0}$ are peak values.

${ }^{\mathrm{b}}$ The value of $\Delta m$ is chosen at $k a=3$ which is probably an overestimate. This means that the ratios in this column are perhaps conservatively estimated.

The data of table I show that, as the magnetic field angle $\psi$ increases, the polarization reduction factor for the cylinders is more drastic than that for Rayleigh particles. This is shown in the third column by the fact that the ratio of reduction factors is always less than 1 . On the other hand, the ratio of polarization to extinction as given in the fourth column is greater than the maximum observed value of $\left(\Delta m_{p} / \Delta m\right)_{\max }$ $\equiv 0.065$ (ref. 11) for values of $\psi$ even beyond $\psi=30^{\circ}$. In figure 9 is shown the ratio of polarization to extinction for the oriented cylinders as a function of size $(\mathrm{ka})$. Comparison of these curves with the maximum observed value of this ratio indicates that a size distribution of such

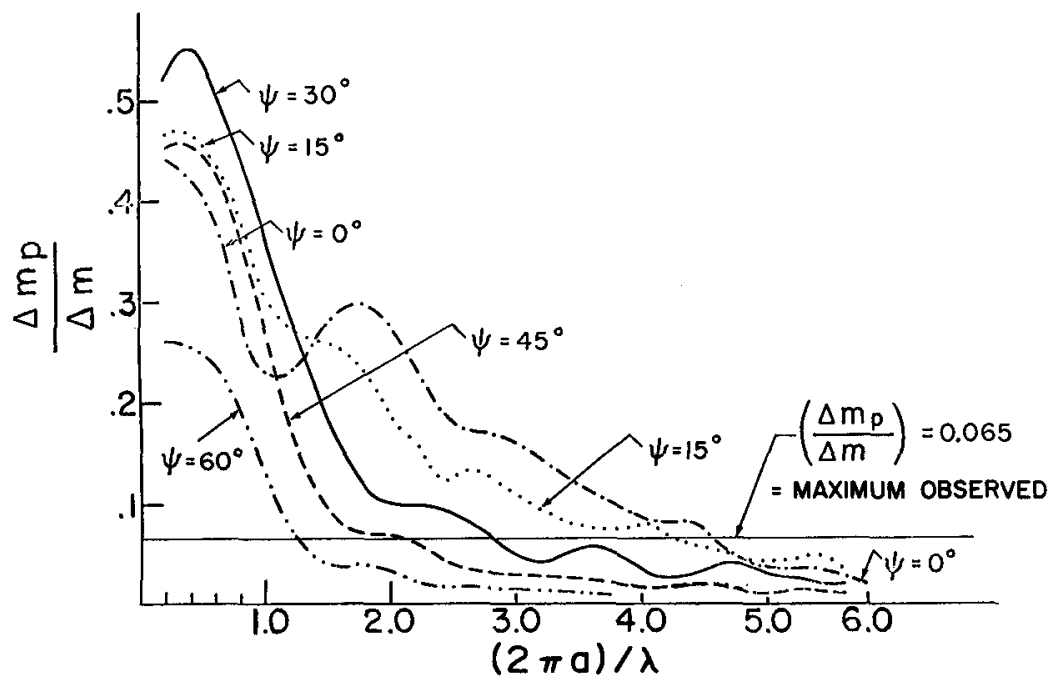

FIGURE 9. - Polarizing capabilities of Davis-Greenstein-oriented cylinders as a function of $(2 \pi a) / \lambda$. The horizontal line is the maximum observed polarizing capability of interstellar grains. 
grains would be able to produce sufficient polarization. Furthermore, the calculated ratio of polarization to extinction is apparently sufficiently large at the smaller angles that one may well tolerate relatively incomplete orientation (as given by letting the $B$ direction be contained within some cone) and still achieve a rather high degree of polarization. Because more detailed calculations of this averaging are in process, further discussion of this point is deferred

\section{CONCLUDING REMARKS}

On the basis of the limited theoretical model of a cloud of interstellar grains consisting of elongated particles (infinite dielectric cylinders) of a single size, some hitherto unexpected correlations between variations in the wavelength dependence of polarization and variations in the wavelength dependence of extinction have been demonstrated. This investigation, although only preliminary, represents the first completely self-consistent theoretical approach to two of the manifestations of interstellar grains-extinction and polarization.

Calculations are either in progress or being planned that will take into account wavelength dependence of index of refraction (including absorption), distribution of particle sizes, variations in particle size dis-

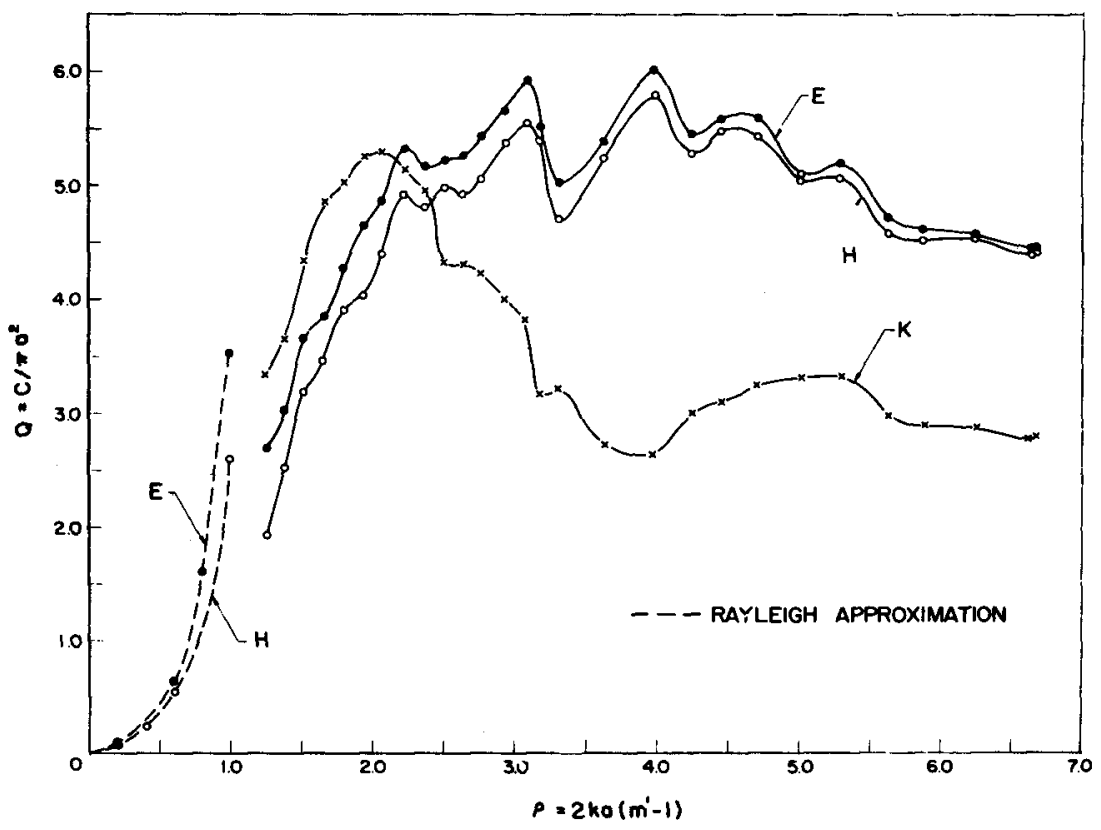

FIGURE 10. - Extinction efficiencies for three orthogonal orientations of prolate spheroids. $b / a=2.0 ; m=1.33-0.05 i$. 
tribution, and incomplete Davis-Greenstein orientation. It is within the realm of possibility to uncouple the extinction and polarization through different clouds along the line of sight via the effect of variations of polarization angle with wavelength. (See refs. 12 and 13.)

Of the many questions which need to be considered, there is one which does not exhibit itself in the single size model but which is quite important when one has a distribution of sizes. This question is the distribution of elongations with particle size. Basically, the answer to this question lies in the physics and chemistry of grain growth. Phenomenologically it exhibits itself in determining the wavelength dependence of

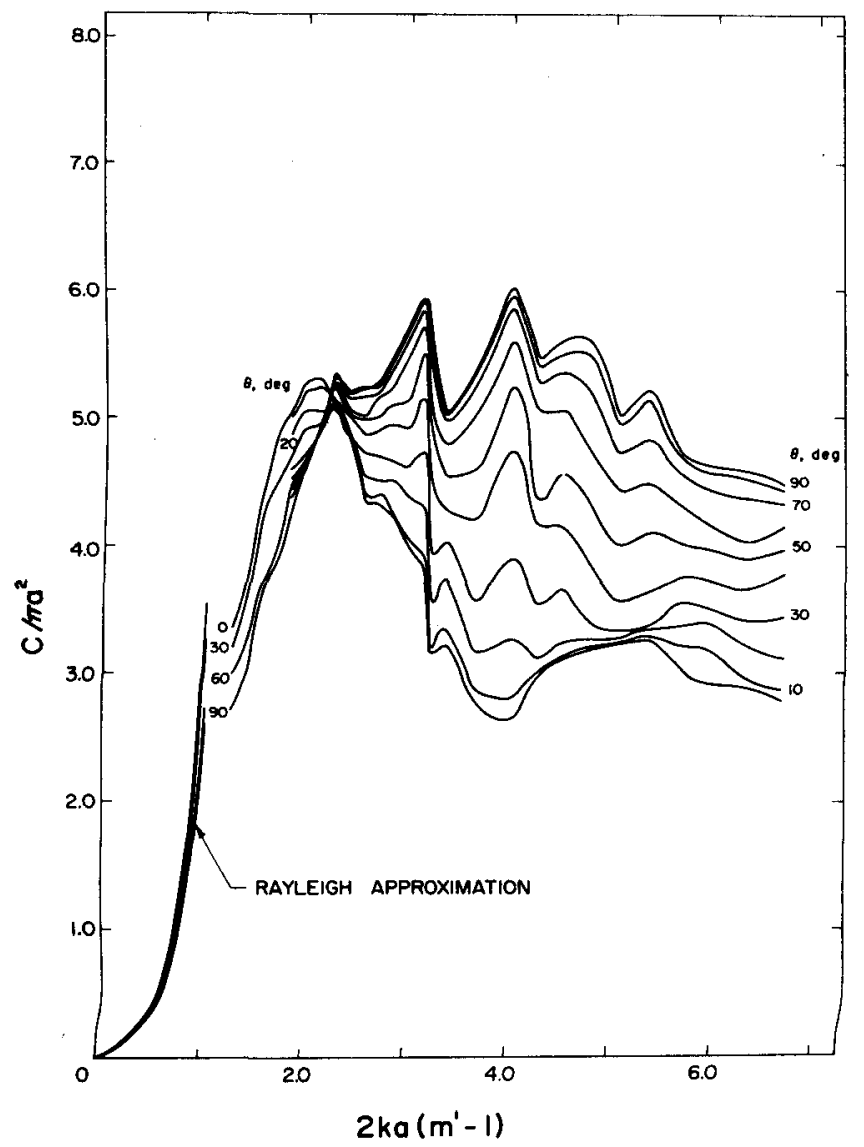

FIGURE 11. - Extinction efficiencies for prolate spheroids whose symmetry axes are tilted in the plane containing electric and propagation vectors of incident radiation.

Tilt angle of $90^{\circ}$ corresponds to case $E$ of figure 10 . Tilt angle of $0^{\circ}$ corresponds to case $K$ of figure 10. $b / a=2.0 ; m=1.33-0.05 i ; E$ plane. 


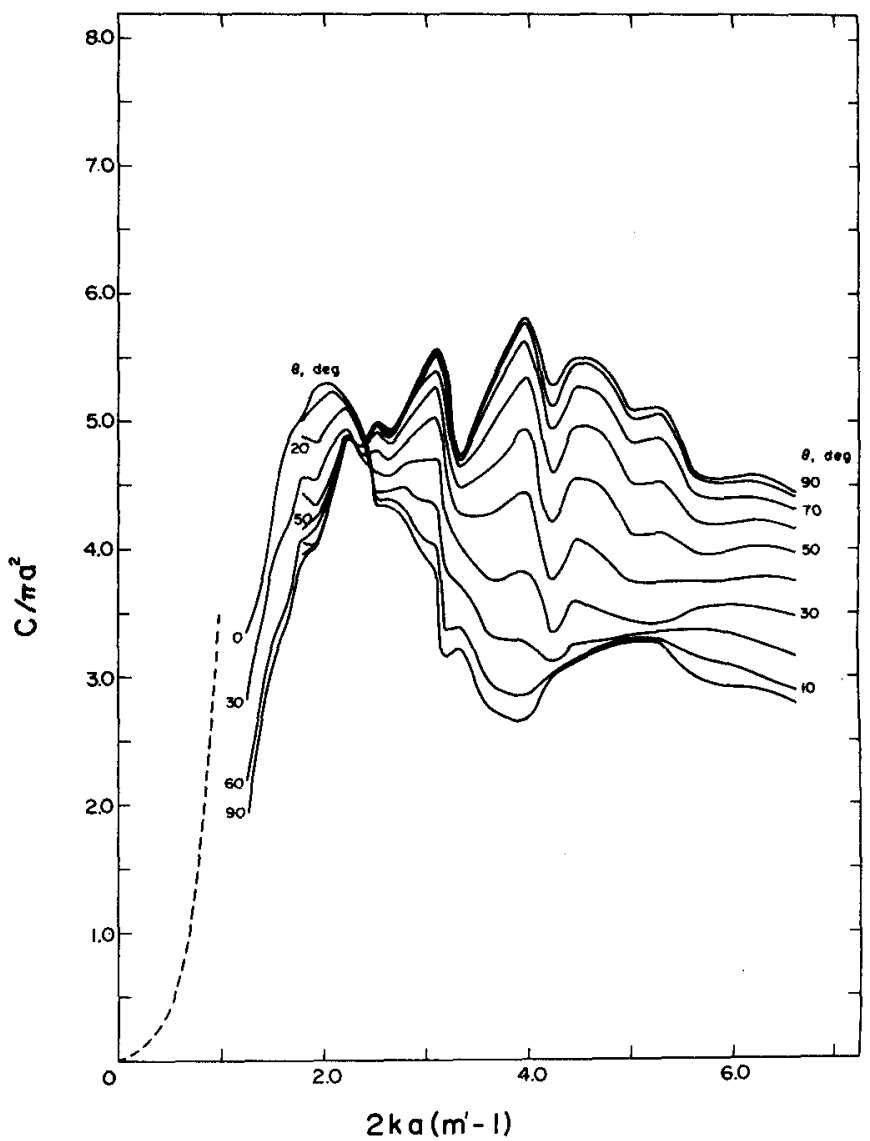

FIGURE 12. - Extinction efficiencies for prolate spheroids whose symmetry axes are tilted in the plane containing magnetic and pronagation vectors of incident radiation.

Tilt angle of $90^{\circ}$ corresponds to case $H$ of figure 10.

Tilt angle of $0^{\circ}$ corresponds to case $K$ of figure 10. $b / a=2.0 ; m=1.33-0.05 i ; H$ piane.

extinction and polarization. Initially two extremes will be considered; namely, that in which all particles either have the same elongation or the same length.

Finally, with the improved microwave scattering techniques now available (ref. 14) the finite particle model will be subjected to detailed scrutiny in the near future. Figures 10 to 12 show some results already obtained for arbitrarily oriented prolate spheroids.

\section{REFERENCES}

1. Platt, J.: On the Optical Properties of Interstellar Dust Astrophys. J., vol. 123, 1956, p. 486.

2. Greenberg, J. M.: Interstellar Grains. Ann. Rev. Astr. and Ap., vol. 1, 1963, p. 267. 
3. GeHRELS, T.: Astron. J., vol. 65, 1960, p. 466.

4. Greenberg, J. M.; Lind, A. C.; Wang, R. T.; and Libelo, L. F.: The Polarization of Starlight by Oriented Non-Spherical Particles. Electromagnetic Seattering. Proceedings of ICES I (New York), Pergamon Press, 1963, p. 123.

5. Lind, A. C.; and GreenberG, J. M.: Electromagnetic Scattering by Obliquely Oriented Cylinders. J. Appl. Phys., vol. 37, 1966, pp. 3195-3203.

6. Greenberg, J. M.; Lind, A. C.; Wang, R. T.; and Libelo, L. F.: Scattering by Nonspherical Particles. Proceedings of ICES II (Amherst, Mass.), 1965.

7. Davis, L., Jr.; and Greenstein, J. L.: The Polarization of Starlight by Aligned Dust Grains. Astrophys. J., vol. 114, 1951, p. 206.

8. VAN De Hulst, H. C.: Light Scattering by Small Particles. John Wiley \& Sons, Inc., 1957.

9. Greenberg, J. M.; and Meltzer, A. S.: The Effect of Orientation of Non-Spherical Particles on Interstellar Extinction. Astrophys. J., vol. 132, 1960, p. 660.

10. Wilson, R.: The Relation Between Interstellar Extinction and Polarization. Roy. Astron. Soc., Monthly Notices, vol. 120, 1960, p. 51.

11. Schмid', T.: Zur Analyse des Zusammenhangs zwischen interstellarer Polarisation und interstellarer Verfärbung. Zs. Astrophys., vol. 46, 1958, p. 145.

12. Gehrels, T.; and Silvester, A. B.: Wavelength Dependence of Polarization V. Position Angles of Interstellar Polarization. Astron. J., vol. 70, 1965, p. 579.

13. Treanor, P. J.: Wavelength Dependence of Interstellar Polarization. Astron. J., vol. 68,1963, p. 185.

14. Lind, A. C.; Wang, R. T.; and Greenberg, J. M.: Microwave Scattering by NonSpherical Particles. Appl. Opt., vol. 4, 1965, p. 1555.

\section{DISCUSSION}

Wickramasinghe: Do those extinction curves go up at all in the far ultraviolet? Is there a point of inflection in the curve after which the slope increases? Are they concave upward?

Greenberg: For sufficiently small values of $(2 \pi a) / \lambda$ they are concave upward.

Wickramasinghe: Is the abscissa $\lambda^{-1}$ ?

Greenberg: This abscissa is $(2 \pi a) / \lambda$.

Wickramasinghe: Did you make the computations for a particular value of the radius in order to compare the result with the observed extinction curve?

Greenberg: You could say that we made computations for a particular radius and then let the wavelength vary, but since we used a constant index of refraction, it doesn't make any difference.

Wampler: Did I understand you correctly that, for small-diameter infinite cylinders, the direction of the polarization vector can change with the wavelength?

Greenberg: Yes, but only for orientations such that the particle axis is directed close to the direction of the radiation.

Wampler: I think the case for a conducting particle is different in that you would get different polarization with wavelength because of plasma oscillations in the particle. 
Greenberg: It is true that a reverse polarization results if a particle is too large. If the particle is very small compared with the wavelength, the polarization is just as you would intuitively predict (except for certain orientations). If the particle is about the size of the wavelength or bigger, whether dielectric or metallic, then reversals of polarization occur. But at that point, in general, the polarization is very small; in fact, it is approaching zero. In the limit, since the wavelength is small compared with the size, the polarization is identically zero.

Wampler: Do you have a feeling about whether this would be observable if a normal Oort-van de Hulst size distribution is assumed?

Greenberg: In the case we have presented here, we have not reached the point of reversal in any of the particle sizes.

Nandy: Do you still believe that the longitudinal dependence of the extinction law is a test to discriminate whether the particles are dielectric or not?

Greenberg: If all other effects could be eliminated it would be a legitimate criterion. However, even that criterion would have to be quantitative. I would expect that the wavelength dependence of extinction by particles which are anisotropic in optical and shaped properties such as graphite would also exhibit variations in the wavelength dependence of extinction because of orientation. However, I think that these differences in the ultraviolet would not be as large for anisotropic as for dielectric particles. There may be significant but small, differences in the visible.

Wickramasinghe: In matching these experimental curves does one have to use a very special size in order to get the peak of the polarization curve to fit?

Greenberg: I use the Oort-van de Hulst size distribution.

Wickramasinghe: Yes, but you nevertheless have to choose a size parameter so that the peak comes in the right place.

Greenberg: Yes, but I don't think the peak is that well defined, because if $I$ shift by 5 percent I get a small shift in the peak. But you are saying that the peak is sensitive to the size. I agree; but it is not as sensitive to the size parameter in a size distribution as it is to a single size. That's a very important point. For a single size, a shifting in size by 5 percent shifts the peak by 5 percent. However, for a size distribution, various particles in the size distribution give a different contribution. Therefore, even if we took a larger size distribution, the smaller particles would still give relatively larger contributions and the wavelength dependence would not be modified by the amount in which the parameter in the Oort-van de Hulst type of size distribution is changed.

Gehrels: Suppose for figure 8 you had assumed the Oort-van de Hulst distribution to peak at $2 a=0.3$ instead of at $2 a=0.6$. Have you tried this?

Greenberg: No. 
Gehrels: You might have gotten a slightly better fit.

Greenberg: It wouldn't have shifted by a factor of 2 , but it would have shifted the peak toward the shorter wavelength.

Gehrels: That is just where your fit is deficient.

Greenberg: That is possible. I can't say how much of a modification I would need.

Gehrels: Why did van de Hulst consider the ratio of polarization to extinction insufficient in the calculations?

Greenberg: The basis for doubting dielectric particles as sufficiently good polarizers was founded on insufficient knowledge of the dependence of polarizing ability on (1) particle elongation; (2) imperfect orientation, i.e., Davis-Greenstein spinning type (ref. 7) as a function of perfect ful! (picket-fence) alinement; and (3) incomplete orientation of the spin axis of the particles. Let me summarize here the pertinent results contained in reference 4.

Let $C_{E}$ and $C_{H}$ be cross sections for particles whose symmetry axis is perpendicular to the direction of propagation of the radiation and parallel to the electric and magnetic fields $(E$ and $H$ ), respectively, of plane-polarized radiation. By $C_{E(x)}$ and $C_{H(X)}$ we denote the cross sections when the symmetry axis makes an angle $\chi$ with respect to the propagation direction.

With regard to elongation, in the range of particle sizes relative to wavelength characteristic of the visual spectrum, the average ratio $\left(C_{E} / C_{H}\right)$ for prolate spheroids of elongation 2 and index of refraction $m=1.33-0.05 i$ is the same as that for infinitely long cylinders with index of refraction $m=1.30$; namely, $\left(C_{E} / C_{H}\right) \approx 1.16$. Clearly, finite elongated particles may well be as effective as infinitely elongated particles in polarizing radiation.

In the matter of orientation, the ratio

$$
R_{\text {D.G. }}=\frac{\int_{0}^{\pi}\left[C_{E}(\chi)-C_{H}(\chi)\right] \mathrm{d} \chi}{\frac{1}{2} \int_{0}^{\pi}\left[C_{E}(\chi)+C_{H}(\chi)\right] \mathrm{d} \chi}
$$

is, for a single particle size, equal to the ratio of polarization to extinction $\Delta m_{p} / \Delta m$ for perfect spinning orientation. For picket-fence orientation, this ratio is

$$
R_{\text {P.F. }}=\frac{C_{E}-C_{H}}{\frac{1}{2}\left(C_{E}+C_{H}\right)}
$$

Averaging both of these quantities yields $R_{\text {P.F. }} \approx 0.15$ and $R_{\text {D.G. }} \approx 0.09$, a reduction by a factor of 0.6 .

Concerning partial Davis-Greenstein orientation, if the angle between 
the spin axis (normal to symmetry axis) and the direction of propagation is $\psi$, the ratio of polarization to extinction is reduced by the factor (approximate) $\cos 2 \psi$. If the spin axes are distributed within this angle, then the reduction is somewhat less. Noting that the maximum observed ratio of polarization to extinction is $\Delta m_{p} / \Delta m \cong 0.6$ and combining this with $R_{\text {D.G. }} \cong 0.09$, one finds an allowable half-angle $\psi \approx 20^{\circ}$ to $30^{\circ}$ for the cone containing the spin axes.

Wickramasinghe: One should really remember that the grains are spinning in cones. They are all alined about some direction, only to the extent of spinning in small cones about this direction.

Greenberg: I considered the worst possible case. If I put them within a cone, they are going to give a higher polarization than the case considered by us.

Wickramasinghe: But isn't it more realistic to take the case where the alinement has some sort of statistical effect? Then you would get out of one of the difficulties that van de Hulst was worrying about.

Greenberg: No, that is not what he was worried about; he was worried about getting the polarization. If $I$ get this with random mag. netic-field orientation, this is equivalent to some sort of a distribution in cone angle.

Behr: If we go down in the $\lambda^{-1}$ direction, the cross sections in the $H$-plane and $E$-plane will go down to zero. Is the ratio also going to zero?

Greenberg: As a matter of fact, the ratio will approach a finite limit. 